\title{
Electric-Field Sensing with a Scanning Fiber-Coupled Quantum Dot
}

\author{
D. Cadeddu, ${ }^{1}$ M. Munsch, ${ }^{1}$ N. Rossi, ${ }^{1}$ J.-M. Gérard, ${ }^{2}$ J. Claudon, ${ }^{2}$ R. J. Warburton, ${ }^{1}$ and M. Poggio ${ }^{1}$ \\ ${ }^{1}$ Department of Physics, University of Basel, 4056 Basel, Switzerland \\ ${ }^{2}$ Univ. Grenoble Alpes, CEA, INAC, PHELIQS, "Nanophysique et semiconducteurs" Group, \\ F-38000 Grenoble, France
}

(Received 5 May 2017; revised manuscript received 20 July 2017; published 29 September 2017)

\begin{abstract}
We demonstrate the application of a fiber-coupled quantum dot (QD) in a tip as a scanning probe for electric-field imaging. We map the out-of-plane component of the electric field induced by a pair of electrodes by the measurement of the quantum-confined Stark effect induced on a QD spectral line. Our results are in agreement with finite-element simulations of the experiment. Furthermore, we present results from analytic calculations and simulations which are relevant to any electric-field sensor embedded in a dielectric tip. In particular, we highlight the impact of the tip geometry on both the resolution and sensitivity.
\end{abstract}

DOI: $10.1103 /$ PhysRevApplied.8.031002

Motivated by a desire to measure the electronic properties of surfaces and nano-objects, many nanoscale electric-field sensors have been developed over the years. Electrostaticforce microscopy [1,2], scanning Kelvin probe force microscopy [3], sensing based on nitrogen-vacancy centers in diamond [4], and scanning single-electron transistors (SETs) [5,6] have already established themselves as sensitive electric-field detectors reaching subelementary charge sensitivity and subnanometer spatial resolution. The most sensitive of these devices, the scanning SET, typically operates below $1 \mathrm{kHz}$ due to the high intrinsic resistance of the SET and the capacitive load of the leads. This slow operation speed also exposes the sensor to $1 / f$ charge noise, which limits its sensitivity to $10^{-4} e / \sqrt{\mathrm{Hz}}$ within $100 \mathrm{~nm}$ of the probe [7]. Although radio frequency SETs achieve bandwidths in excess of $100 \mathrm{MHz}$ and 100 times better sensitivity than conventional SETs [8], so far they have been realized only as on-chip electrometers not amenable to spatial scanning [9].

In 2015, Wagner et al. used a noncontact atomic force and scanning tunneling microscope functionalized with a single molecule to image the dipole field of an adatom on a surface [10]. This first demonstration of scanning quantumdot microscopy (SQDM) registered single-electron charging events of a molecular quantum dot (QD) to produce three-dimensional images of the local electrostatic potential with subnanometer resolution. In transport experiments, gate-defined QDs are employed as single-charge detectors $[11,12]$, and self-assembled QDs are employed as all-optical electrometers, demonstrating a sensitivity of $5(\mathrm{~V} / \mathrm{m}) / \sqrt{\mathrm{Hz}}$ [13]. These kind of QDs are also used to determine the position of single-defect charges within $100 \mathrm{~nm}$ of a QD with a precision of $5 \mathrm{~nm}$ [14]. Electric fields in QDs produce large Stark shifts, which, due to a built-in electric dipole, are nearly linear around zero field. Nevertheless, a scanning electric-field sensor based on an optically active semiconductor QD has not yet been realized. Such SQDM has the potential for a very large bandwidth, which, unlike electronic and mechanically addressable sensors, is limited only by the spontaneous emission rate of the QD and could therefore approach the gigahertz range.

Here we show the proof-of-principle application of an optical fiber-coupled semiconductor QD as a scanning electric-field sensor. By tracking the induced energy shift on the peak of a single transition of a QD, we map the vertical component of an external applied field. Our device is composed of a self-assembled InAs QD located in the tip of a fiber-coupled GaAs photonic wire. The QD emits preferentially into the waveguide mode, which expands adiabatically through a tapering of the photonic wire and ensures good outcoupling [15]. The design of the photonic wire ensures both efficient guiding of the QD fluorescence as well as proximity of the QD to the sample, which is a necessary condition for scanning probe microscopy. With the help of numerical simulations, we also investigate the unavoidable perturbation of the external field due to the dielectric nature of the probe, pointing toward geometric improvements to reduce this effect and increase the sensitivity of the device.

The photonic trumpet used here is obtained through an etching process carried out on a GaAs wafer containing a layer of self-assembled InAs QDs grown by molecular beam epitaxy [15]. The layer of QDs is located at $z_{\mathrm{dot}}=110 \mathrm{~nm}$ above the bottom facet. The Gaussian intensity profile obtained at the top facet of the $11-\mu \mathrm{m}$-tall wire structure enhances the coupling of the QD emission directly into the single-mode fiber [16]. We select a wire with a top diameter of $1.64 \mu \mathrm{m}$ and a bottom diameter $b=350 \mathrm{~nm}$. The wire is then cleaved from the substrate under an optical microscope using a micromanipulator. Using the same apparatus, it is then transferred and glued to the core of a single-mode optical fiber. Optimal coupling is obtained by centering the wide top facet of the photonic wire onto the core of the fiber [17], as shown in Fig. 1(a). The final device constitutes a reliable fiber-coupled source of single photons with a photon collection efficiency of approximately $6 \%$, which is an order 

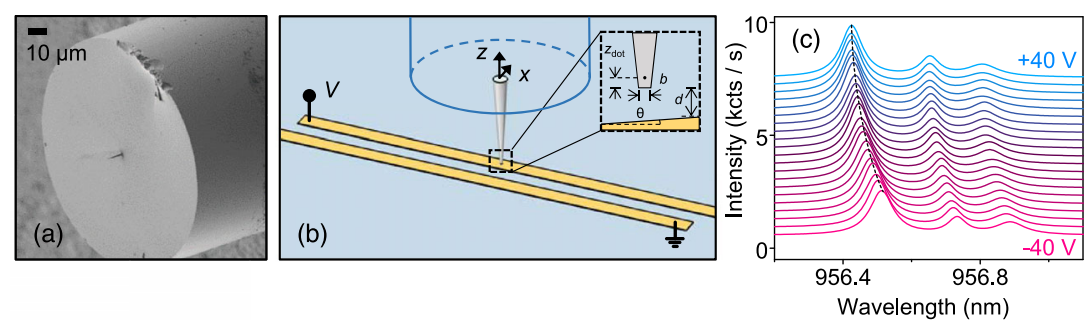

FIG. 1. Fiber-coupled QD electrometer. (a) Scanning electron micrograph of the fibercoupled photonic wire. (b) Schematic of the measurement setup. Inset: Cross section of the $x z$ plane. (c) Lorentzian fits of the PL spectrum showing excitonic lines associated with one QD for different values of $V$ and $d=10 \mathrm{~nm}$. The spectra are offset for clarity. of magnitude better than directly coupling the fiber to a QD in bulk GaAs.

In order to investigate the performance of our probe as a sensor of an electric field, we mount it in a low-temperature scanning probe microscope. The QDs at the end of the photonic wire are excited nonresonantly with a cw diode at $830 \mathrm{~nm}$, which excites carriers directly in the wetting layer and avoids heating of the GaAs wire [17]. Photoluminescence (PL) from the QDs is guided into the fiber by the photonic wire and analyzed with a spectrometer equipped with a CCD camera. The fiber-coupled photonic tip is then positioned over one of two parallel Au electrodes, to which we apply the voltage $V$ while the other is grounded, as shown in Fig. 1(b). The tip-sample distance $d$ is set to $10 \mathrm{~nm}$ from the gate surface with zero defined by the quenching of PL from the QD, most likely due to strain induced by contact. This distance and the lateral position of the tip are controlled by piezoelectric scanners without feedback to stabilize the position. The electrodes, shown schematically in Fig. 1(b), are deposited on a $\mathrm{Si} / \mathrm{SiO}_{2}$ substrate and are $80 \mathrm{~nm}$ thick, $2 \mu \mathrm{m}$ wide, and $2 \mu \mathrm{m}$ apart from each other. The resulting electric field tilts the energy bands of the semiconductor, and, due to the quantum-confined Stark effect [18,19], the transition energies of each QD are shifted to lower energy. Here we focus on a bright peak centered at $956.4 \mathrm{~nm}$ with a linewidth of $100 \mu \mathrm{eV}$, which we attribute to an excitonic transition in a single QD. By sweeping the applied voltage $V$ from -40 to $+40 \mathrm{~V}$, we observe a shift in the energy of the emitted PL that is well described by a quadratic function of the applied voltage - and therefore of the applied electric field—as shown in Fig. 1(c):

$$
\xi=\xi_{0}-p_{\|} E_{\|}+\beta_{\|} E_{\|}^{2}+\beta_{\perp} E_{\perp}^{2},
$$

where $\xi_{0}$ is the unperturbed energy and $p_{\|}$is the static electric dipole of the QD exciton parallel to the wire axis. This axis coincides with the QD growth direction. Because of the inplane symmetry of the QDs, there is no dipole term perpendicular to the axis $[20,21] . \beta_{\|}\left(\beta_{\perp}\right)$ and $E_{\|}\left(E_{\perp}\right)$ are the polarizability of the QD exciton and the applied electric field, parallel (perpendicular) to the wire axis, respectively.

The presence of the photonic wire, due to its dielectric nature, reduces the field at the QD position and significantly perturbs the external applied field. In the simplest approximation, a thin dielectric cylinder with a uniform and unidirectional polarization strongly suppresses electric fields applied perpendicular to its long axis while leaving parallel fields largely unchanged. An analytic model of a conical section matching our photonic trumpet geometry, as well as a more realistic finite-element calculation, show that the wire's narrow radial cross section results in an efficient screening of $E_{\perp}$, while $E_{\|}$is less affected [22]. At the QD position $z_{\text {dot }}=110 \mathrm{~nm}$ above the bottom facet, this directional screening effectively projects the unperturbed electric field along $\hat{z}$. The magnitude of the effect depends on the diameter of the facets, the length of the wire, the position of the QD, and the direction of the field with respect to the long axis of the wire. This screening, as well as a distortion of the electric field outside the photonic tip, is shown in simulations of our experimental geometry in Fig. 2. In our experiment, the field at the QD position reaches values of $60 \%$ and $20 \%$ of the applied external field in the $\hat{z}$ and $\hat{x}$ direction, respectively.

We map the spatial dependence of the electric field produced by the gates by scanning the photonic wire tip and sweeping the applied voltage at every position. Note that the electric field at the QD location $\vec{r}$ is the sum of two distinct contributions $\vec{E}(\vec{r}, V)+\delta \vec{E}$, where the first term is due to the voltage applied across the electrodes and the second term is due to charges trapped in the vicinity of the QD. $\vec{E}(\vec{r}, V)=\vec{\alpha}(\vec{r}) \cdot V$, where $\vec{\alpha}(\vec{r})$ is a position-dependent proportionality constant, which describes the spatial configuration of the electric field produced by the split gates and the dielectric wire. This term is a single-valued function of $\vec{r}$ and $V$. In contrast, $\delta \vec{E}$ is not a single-valued function of $\vec{r}$ or $V$; it describes an electric field that changes upon the stochastic reorganization of charges near the QD. These

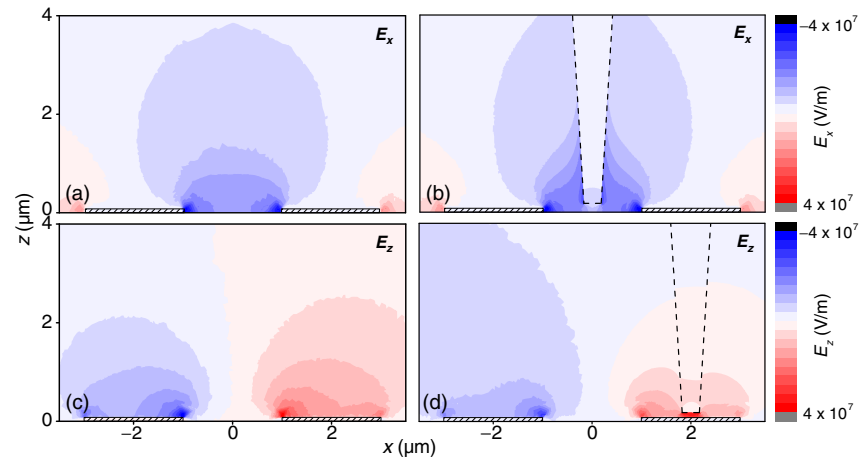

FIG. 2. Simulated maps of $\vec{E}(\vec{r})$. The components (a) $E_{x}$ and (c) $E_{z}$ are generated by the pair of electrodes. The left electrode is grounded, and the right one is held at $V=40 \mathrm{~V}$. (b) and (d) show the same electric-field components in the presence of the dielectric tip. Note the pronounced screening of $E_{x}$ by the photonic tip compared to that of $E_{z}$ at the position of the QD near the apex of the probe. 

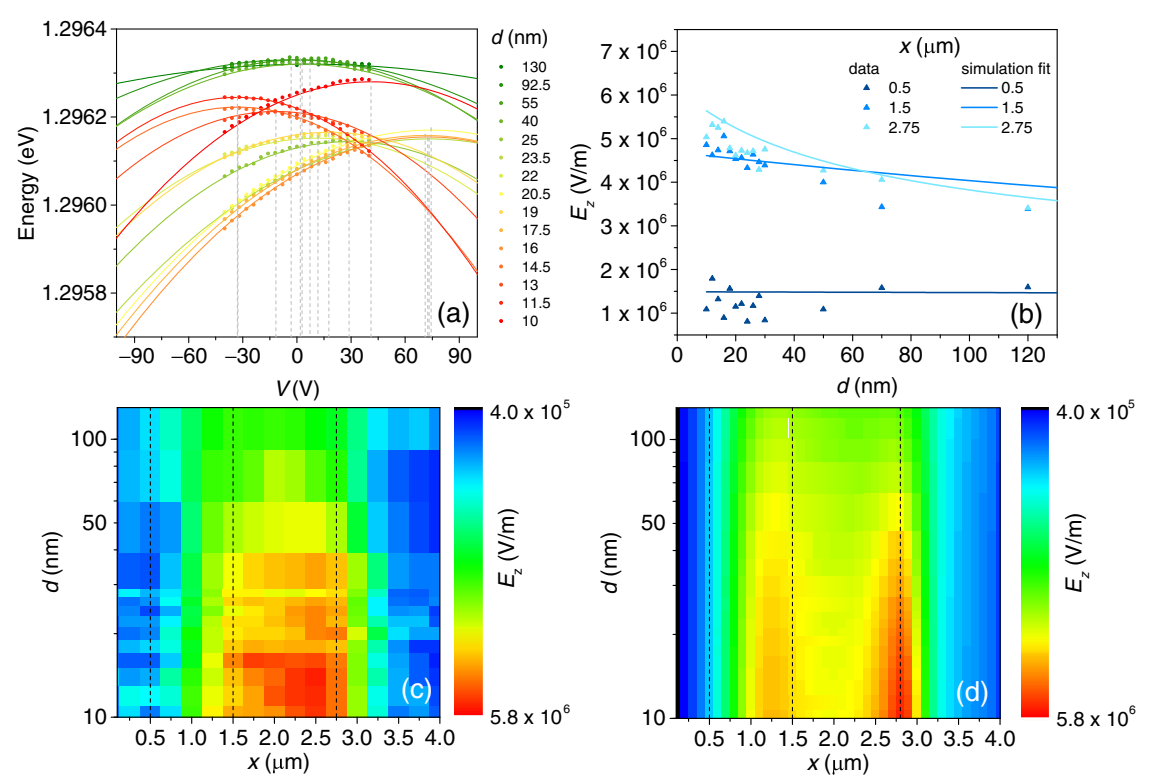

FIG. 3. Experimental results. (a) Energy of the leftmost QD emission peak in Fig. 1(c) vs the applied voltage $V$ for different values of tip-electrode distance $d$. Solid lines are parabolic fits, and gray dashed lines indicate the vertices of each parabola. The tip is positioned at the center of the electrode $(x=2 \mu \mathrm{m})$. (b) Triangles represent $E_{z}$ as a function of $d$ at $V=40 \mathrm{~V}$ extracted from the curvature fits for different $x$ positions; solid lines show corresponding simulated values of $E_{z}$. Full maps of $E_{z}$ as a function of $x$ and $d$ at $V=40 \mathrm{~V}$ extracted from (c) measurements and (d) simulations. Simulations consider a $z_{\text {dot }}=110 \mathrm{~nm}$ with $b=$ $350 \mathrm{~nm}$ and a tilt angle $\theta=5^{\circ}$. Black dashed lines correspond to the line cuts in (b). In both (b) and (c), each point requires $21 \mathrm{~s}$ of measurement time; the full map in (c) requires $68 \mathrm{~min}$. sudden, but infrequent, rearrangements occur on time scales on the order of a single voltage scan or longer and are often induced by the rapid changes in voltage or position occurring between scans.

In our geometry, $E_{\|}=E_{z}+\delta E_{z}$ and $E_{\perp}=$ $\sqrt{\left(E_{x}+\delta E_{x}\right)^{2}+\left(E_{y}+\delta E_{y}\right)^{2}}$. We scan above one electrode, where the electric field points nearly exclusively along $\hat{z}$. Given the direction and the preferential penetration of the field in the $\hat{z}$ direction, we can assume $E_{x}$ to be negligible in this region. $E_{y}$ vanishes due to the symmetry of the electrode structure. As a result, (1) becomes

$$
\begin{aligned}
\xi= & {\left[\xi_{0}-p_{\|} \delta E_{z}+\beta_{\|} \delta E_{z}^{2}+\beta_{\perp}\left(\delta E_{x}^{2}+\delta E_{y}^{2}\right)\right] } \\
& -\left[p_{\|}-2 \beta_{\|} \delta E_{z}\right] E_{z}+\beta_{\|} E_{z}^{2} .
\end{aligned}
$$

Note that both the constant and linear terms in $E_{z}$ (the first two terms in square brackets) depend on the electric field due to local charge reorganization. These terms are therefore subject to random and infrequent shifts. The quadratic term, on the other hand, depends only on the polarizability along the wire axis.

By collecting PL spectra, we measure the dependence of $\xi$ on both voltage and position in the $x z$ plane. The measured QD exciton energies show a parabolic dependence on $V$ with an offset $a_{0}$, a linear coefficient $a_{1}$, and a curvature $a_{2}$, each depending on the position in the $x z$ plane, as seen in Fig. 3(a). As expected from the dependence of the constant and linear terms in (2) on components of the stochastic field $\delta \vec{E}, a_{0}$ and $a_{1}$ appear random and are observed to be hysteretic in both voltage and position. They are likely determined by the charging and discharging of defects within the photonic wire, which generate an extra electric field in the vicinity of the QD. On the other hand, $a_{2}$ remains constant as a function of voltage and reproducible as a function of the position, following what is expected from (2): $a_{2}(x, z)=\beta_{\|} \alpha_{z}(x, z)^{2}$, where $\beta_{\|}$is a constant and $\alpha_{z}(x, z)$ is set by the configuration of the electrodes.

In order to make a detailed comparison to the experiment, we make a finite-element simulation of $\alpha_{z}(x, z)$ at the position of the QD as the photonic wire is scanned above the electrode. A corresponding experimental map of this term can be extracted from the fits to the measured data, since $\alpha_{z}(x, z)=\sqrt{a_{2}(x, z) / \beta_{\|}}$. In Figs. 3(c) and 3(d), we plot the measured and simulated $E_{z}(x, z)$, respectively, corresponding to an applied voltage of $V=40 \mathrm{~V}$. In order to match the spatial dependence of our measurements to the simulations, we introduce a tilt angle $\theta=5^{\circ}$, as shown in the inset in Fig. 1(b). Such a misalignment between both scanning stages is experimentally reasonable and, in practice, difficult to avoid. A polarizability $\beta_{\|}=-0.012 \pm$ $0.005 \mu \mathrm{eV} /(\mathrm{kV} / \mathrm{cm})^{2}$ brings the measured and simulated values of $E_{z}(x, z)$ into numerical agreement, as shown in Figs. 3(b) and 3(c). Such a polarizability is an order of magnitude smaller than what is typically observed in the literature for single-exciton transitions in similar QDs under resonant excitation [23-25]. Under nonresonant excitation-as in this case-a variety of groups have observed reduced Stark shifts [26], likely due to the screening of electric fields by the clouds of electron-hole pairs generated around the QD.

The similarity of the measured and simulated maps of $E_{z}(x, z)$ demonstrates the successful implementation of our QD probe to spatially map the magnitude of a dc electric field along one direction. Nevertheless, the simulation does not capture all of the details of the measured map, indicating that the experiment is still affected by charging effects not included in our model. Note that, although we fit to full voltage sweeps at each position to extract the electric-field configuration, one could reduce the noise and speed up the measurement by applying a small ac voltage to the gate and recording the QD's response. Energy shifts at $f$ and $2 f$, where $f$ is the frequency of the applied field, would correspond to 


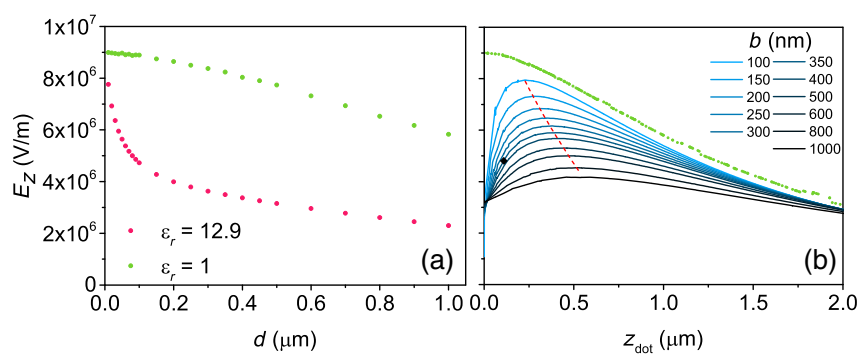

FIG. 4. Influence of the tip geometry on sensing capabilities. (a) Simulated $E_{z}$ at $z_{\text {dot }}=110 \mathrm{~nm}$ is plotted in pink as a function of tip-electrode distance $d$ in a GaAs tip with $b=350 \mathrm{~nm}$. The corresponding condition without screening is plotted in green. (b) $E_{z}$ values along a $z$ line cut with the photonic tip at $d=$ $100 \mathrm{~nm}$ for different values of the bottom diameter $b$. $z_{\text {opt }}$ is the position where $E_{z}$ is maximum inside the tip and is shown by the red dashed line for different values of $b$. The black diamond indicates the QD position in our current device. The green dots are values of $E_{z}$ in the absence of the trumpet, as a reference.

the linear contribution, arising from the electric dipole of the $\mathrm{QD}$, and to the quadratic contribution, arising from the polarizability, respectively [27]. Similarly, static electric fields generated by arbitrary samples without electrodes could be mapped by dithering the position of the QD sensor at frequency $f$ and measuring the corresponding response. As a result, one could measure the spatial derivative of $E_{z}$ along the direction of the dither. In this way, the technique could be generally applied to a variety of samples producing electric fields, including by charge defects on surfaces.

Figs. 3(b) and 3(c) show a rapid decrease in the measured electric field as a function of tip-electrode distance $d$ compared to that expected in a vacuum. As demonstrated in Fig. 4(a), this effect is a direct consequence of the polarization charge induced on the bottom facet of the photonic wire, which screens the out-of-plane electric field impinging on the QD. This effect must be considered for any electric-field sensor based on a dielectric scanning probe. In order to reduce the screening effect for $E_{z}$, which decreases the sensitivity of the sensor and distorts the observed field with respect to the unperturbed case, this surface charge density must be minimized. If we exclude replacing the GaAs tip material with one having a dielectric constant closer to the one of the vacuum, the reduction of this screening charge can be achieved by reducing the diameter $b$ of the bottom facet. This sharpening of the scanning probe would also help to increase the screening of $E_{x}$, making the QD Stark shift an even closer measure of $E_{z}{ }^{2}$. In practice, however, $b$ is constrained to values higher than $190 \mathrm{~nm}$ in order to maintain optimal guiding of the QD PL up the photonic wire and into the optical fiber [28]. The choice of $b$ in turn sets a natural minimum tip-sample distance $d$ of the same order, below which both the spatial resolution will not improve and the tip will strongly perturb the local electric field. Once $b$ and $d$ are fixed, finite-element calculations show that there exists an optimal position for the QD above the bottom facet $z_{\mathrm{opt}}$, as shown by Fig. 4(b). This position minimizes the screening effect and hosts the largest measurable field from the electrodes within the tip. The black diamond in Fig. 4(b) indicates the position of the QD within the device used in our experiments. For $d=100 \mathrm{~nm}$, optimization of $b$ and of $z_{\text {dot }}$ should allow the measurement of weaker fields parallel to the wire axis with almost no tip-induced perturbation of this component. Note that, in order to ensure the maximum reflection in the upward direction, $z_{\text {dot }}$ should be a multiple of $\lambda / 2 n$ from the bottom facet, where $\lambda$ is the wavelength of the emitted light and $n$ the effective index of the fundamental guided mode for a diameter $b$. In general, there is a natural trade-off between a scanning probe tip which minimally perturbs the electric field and one that optimally guides the sensor emission.

In conclusion, we demonstrate the application of a fibercoupled semiconductor QD as a scanning probe for electric-field sensing. The probe QD resides at the apex of a sharp GaAs scanning probe, which allows for both an efficient optical collection and a preferential penetration of on-axis electric fields. Despite position-dependent hysteresis and randomness exhibited by the QD's PL energy and electric dipole, its constant polarizability allows for the extraction of the electric-field magnitude at the position of the probe QD. By scanning this sensing element, we map the spatial dependence of the out-of-plane electric field produced by a pair of microfabricated electrodes at $4 \mathrm{~K}$. Given a measured polarizability $\beta_{\|}=-0.012 \mu \mathrm{eV} /(\mathrm{kV} / \mathrm{cm})^{2}$, typical count rate of $2.3 \mathrm{kHz}$, and emission linewidth of $98 \mu \mathrm{eV}$, the measurements demonstrate a sensitivity of $1.4 \times 10^{6}(\mathrm{~V} / \mathrm{m}) / \sqrt{\mathrm{Hz}}$. Although such a sensitivity is sufficient to demonstrate the feasibility of electric-field microscopy with our fiber-coupled QD sensor, it must be improved in order to approach the performance demonstrated by scanning SETs.

Measuring by resonance fluorescence constitutes a first step: The absence of nonresonant excitation should reduce charge noise near the QD [29], allowing the use of the linear Stark effect. Assuming measured values for the count rate, emission linewidth, and a dipole moment $p_{\|} / e=0.03 \mathrm{~nm}$, a linear measurement would already yield a sensitivity of $5.6 \times 10^{4}(\mathrm{~V} / \mathrm{m}) / \sqrt{\mathrm{Hz}}$. This worst-case estimate does not consider that measuring by resonance fluorescence would also eliminate screening of the sensor QD by photoexcited electron-hole pairs and decrease the QD emission linewidth. Furthermore, by optimizing the geometry of the photonic wire tip and the position of the QD, higher optical-collection efficiencies-resulting in higher count rates-could be combined with better electric-field penetration. These additional improvements would lead to a reduced distortion of the unperturbed electric fields and higher sensitivity. If charge noise near the sensor could be reduced to levels achieved by QDs buried in bulk semiconductor material, a linear Stark effect measurement could then approach $20(\mathrm{~V} / \mathrm{m}) / \sqrt{\mathrm{Hz}}$, assuming $p_{\|} / e=0.3 \mathrm{~nm}$ $[13,14]$. This estimate is based on a count rate of $1 \mathrm{MHz}$ and 
an emission linewidth of $5 \mu \mathrm{eV}$, which are observed in shotnoise-limited resonance fluorescence measurements carried out in photonic-trumpet QDs similar to those measured here [30]. Given that the bandwidth of this type of SQDM is determined by the efficiency of the luminescence collection and limited by the QD's spontaneous emission rate, it could approach the gigahertz range. These characteristics, combined with a spatial resolution roughly set by the bottom diameter of the tip, are promising for the mapping of single charges on surfaces, measuring individual tunneling events, and monitoring charging dynamics in few-electron and mesoscopic systems.

\section{ACKNOWLEDGMENTS}

We thank Jean Teissier and Loïc Lanco for discussions and Sascha Martin for technical support. This work is supported by the European Research Council (Grant No. 334767), the Swiss Nanoscience Institute (Project No. P1207), and the National Centre of Competence in Research, Quantum Science and Technology.

[1] Y. Martin, D. W. Abraham, and H. K. Wickramasinghe, High resolution capacitance measurement and potentiometry by force microscopy, Appl. Phys. Lett. 52, 1103 (1988).

[2] L. Gross, F. Mohn, P. Liljeroth, J. Repp, F. J. Giessibl, and G. Meyer, Measuring the charge state of an adatom with noncontact atomic force microscopy, Science 324, 1428 (2009).

[3] A. K. Henning, T. Hochwitz, J. Slinkman, J. Never, S. Hoffmann, P. Kaszuba, and C. Daghlian, Two dimensional surface dopant profiling in silicon using scanning Kelvin probe microscopy, J. Appl. Phys. 77, 1888 (1995).

[4] F. Dolde, H. Fedder, M. W. Doherty, T. Nöbauer, F. Rempp, G. Balasubramanian, T. Wolf, F. Reinhard, L. C. L. Hollenberg, F. Jelezko, and J. Wrachtrup, Electric-field sensing using single diamond spins, Nat. Phys. 7, 459 (2011).

[5] M. J. Yoo, T. A. Fulton, H. F. Hess, R. L. Willett, L. N. Dunkleberger, R. J. Chichester, L. N. Pfeiffer, and $\mathrm{K}$. W. West, Scanning single-electron transistor microscopy: Imaging individual charges, Science 276, 579 (1997).

[6] J. Martin, N. Akerman, G. Ulbricht, T. Lohmann, J. H. Smet, K. von Klitzing, and A. Yacoby, Observation of electron-hole puddles in graphene using a scanning single-electron transistor, Nat. Phys. 4, 144 (2008).

[7] G. Zimmerli, T. M. Eiles, R. L. Kautz, and J. M. Martinis, Noise in the Coulomb blockade electrometer, Appl. Phys. Lett. 61, 237 (1992).

[8] R. J. Schoelkopf, P. Wahlgren, A. A. Kozhevnikov, P. Delsing, and D. E. Prober, The radio-frequency singleelectron transistor (RF-SET): A fast and ultrasensitive electrometer, Science 280, 1238 (1998).

[9] L. Lu, L. Su, J. Sunl, X. Lil, H. Qinl, Z. Ji, and R. Blick, Modeling a radio-frequency single-electron-transistor scanning probe, Jpn. J. Appl. Phys. 53, 085001 (2014).

[10] C. Wagner, M. F. B. Green, P. Leinen, T. Deilmann, P. Krüger, M. Rohlfing, R. Temirov, and F. S. Tautz, Scanning
Quantum Dot Microscopy, Phys. Rev. Lett. 115, 026101 (2015).

[11] C. Barthel, M. Kjærgaard, J. Medford, M. Stopa, C. M. Marcus, M. P. Hanson, and A. C. Gossard, Fast sensing of double-dot charge arrangement and spin state with a radiofrequency sensor quantum dot, Phys. Rev. B 81, 161308 (2010).

[12] C. Arnold, V. Loo, A. Lemaître, I. Sagnes, O. Krebs, P. Voisin, P. Senellart, and L. Lanco, Cavity-Enhanced RealTime Monitoring of Single-Charge Jumps at the Microsecond Time Scale, Phys. Rev. X 4, 021004 (2014).

[13] A. N. Vamivakas, Y. Zhao, S. Fält, A. Badolato, J. M. Taylor, and M. Atature, A Nanometer-Scale Optical Electrometer, Phys. Rev. Lett. 107, 166802 (2011).

[14] J. Houel, A. V. Kuhlmann, L. Greuter, F. Xue, M. Poggio, B. D. Gerardot, P. A. Dalgarno, A. Badolato, P. M. Petroff, A. Ludwig, D. Reuter, A. D. Wieck, and R. J. Warburton, Probing Single-Charge Fluctuations at a GaAs/AlAs Interface Using Laser Spectroscopy on a Nearby InGaAs Quantum Dot, Phys. Rev. Lett. 108, 107401 (2012).

[15] M. Munsch, N. S. Malik, E. Dupuy, A. Delga, J. Bleuse, J.-M. Gérard, J. Claudon, N. Gregersen, and J. Mørk, Dielectric GaAs Antenna Ensuring an Efficient Broadband Coupling between an InAs Quantum Dot and a Gaussian Optical Beam, Phys. Rev. Lett. 110, 177402 (2013).

[16] P. Stepanov, A. Delga, N. Gregersen, E. Peinke, M. Munsch, J. Teissier, J. Mørk, M. Richard, J. Bleuse, J.-M. Gérard, and J. Claudon, Highly directive and Gaussian far-field emission from giant photonic trumpets, Appl. Phys. Lett. 107, 141106 (2015).

[17] D. Cadeddu, J. Teissier, F. R. Braakman, N. Gregersen, P. Stepanov, J.-M. Gérard, J. Claudon, R. J. Warburton, M. Poggio, and M. Munsch, A fiber-coupled quantum-dot on a photonic tip, Appl. Phys. Lett. 108, 011112 (2016).

[18] P. W. Fry, I. E. Itskevich, D. J. Mowbray, M. S. Skolnick, J. J. Finley, J. A. Barker, E. P. O’Reilly, L. R. Wilson, I. A. Larkin, P. A. Maksym, M. Hopkinson, M. Al-Khafaji, J. P. R. David, A. G. Cullis, G. Hill, and J. C. Clark, Inverted Electron-Hole Alignment in InAs-GaAs Self-Assembled Quantum Dots, Phys. Rev. Lett. 84, 733 (2000).

[19] C. Schulhauser, D. Haft, R. J. Warburton, K. Karrai, A. O. Govorov, A. V. Kalameitsev, A. Chaplik, W. Schoenfeld, J. M. Garcia, and P. M. Petroff, Magneto-optical properties of charged excitons in quantum dots, Phys. Rev. B 66, 193303 (2002).

[20] V. Stavarache, D. Reuter, A. D. Wieck, M. Schwab, D. R. Yakovlev, R. Oulton, and M. Bayer, Control of quantum dot excitons by lateral electric fields, Appl. Phys. Lett. 89, 123105 (2006).

[21] M. M. Vogel, S. M. Ulrich, R. Hafenbrak, P. Michler, L. Wang, A. Rastelli, and O. G. Schmidt, Influence of lateral electric fields on multiexcitonic transitions and fine structure of single quantum dots, Appl. Phys. Lett. 91, 051904 (2007).

[22] See Supplemental Material at http://link.aps.org/ supplemental/10.1103/PhysRevApplied.8.031002 for a detailed analysis of the effect of the geometry and configuration of a dielectric tip in a uniform electric field.

[23] R. J. Warburton, C. Schulhauser, D. Haft, C. Schäflein, K. Karrai, J. M. Garcia, W. Schoenfeld, and P. M. Petroff, Giant 
permanent dipole moments of excitons in semiconductor nanostructures, Phys. Rev. B 65, 113303 (2002).

[24] J. H. Prechtel, F. Maier, J. Houel, A. V. Kuhlmann, A. Ludwig, A. D. Wieck, D. Loss, and R. J. Warburton, Electrically tunable hole $\mathrm{g}$ factor of an optically active quantum dot for fast spin rotations, Phys. Rev. B 91, 165304 (2015).

[25] J. J. Finley, M. Sabathil, P. Vogl, G. Abstreiter, R. Oulton, A. I. Tartakovskii, D. J. Mowbray, M. S. Skolnick, S. L. Liew, A. G. Cullis, and M. Hopkinson, Quantum-confined Stark shifts of charged exciton complexes in quantum dots, Phys. Rev. B 70, 201308 (2004).

[26] A. Faraon, A. Majumdar, H. Kim, P. Petroff, and J. Vučković, Fast Electrical Control of a Quantum Dot Strongly Coupled to a Photonic-Crystal Cavity, Phys. Rev. Lett. 104, 047402 (2010).
[27] N. Rossi, F. R. Braakman, D. Cadeddu, D. Vasyukov, G. Tütüncüoglu, A. Fontcuberta i Morral, and M. Poggio, Vectorial scanning force microscopy using a nanowire sensor, Nat. Nanotechnol. 12, 150 (2017).

[28] I. Friedler, C. Sauvan, J. P. Hugonin, P. Lalanne, J. Claudon, and J. M. Gérard, Solid-state single photon sources: The nanowire antenna, Opt. Express 17, 2095 (2009).

[29] A. V. Kuhlmann, J. Houel, A. Ludwig, L. Greuter, D. Reuter, A. D. Wieck, M. Poggio, and R. J. Warburton, Charge noise and spin noise in a semiconductor quantum device, Nat. Phys. 9, 570 (2013).

[30] M. Munsch, A. V. Kuhlmann, D. Cadeddu, J.-M. Gérard, J. Claudon, M. Poggio, and R. J. Warburton, Resonant driving of a single photon emitter embedded in a mechanical oscillator, Nat. Commun. 8, 76 (2017). 